\title{
Besprechungen
}

von

\section{H. Driesch.}

A. v. Tschermak, Allgemeine Physiologie, in zwei Bänden. Erster Band: Grundlagen der allgemeinen Physiologie. 1. Teil: Allgemeine Charakteristik des Lebens. Physikaljsche und chemische Beschaffenheit der lebenden Substanz. Berlin, Julius Springer. 1916. IX u. 281 S. Preis M. 10.-.

Endlich bekommen wir von einem Zoophysiologen die lang ersehnte strenge ^Allgemeine Physiologier, welche sich den botanischen Werken eines SACHS und Pfeffer würdig an die Seite stellen kann. Diese Zeilen sollen nur hinweisen auf das Werk; wenn die nächste, die erste eigentlich biologische Bandhälfte erschienen sein wird, wird mehr und Eingehenderes zu sagen sein. Der vorliegende Halbband bringt eine breite naturphilosophische Einleitung sowie die Physik and Chemie der lebenden Substanz.

- Vor allem wollte ich dem Leser keine doktrinäre Schulmeinung beibringen, sondern ihn zur selbständigen Kritik sowie zur Aufstellung neuer Probleme und Arbeitshypothesen anregen. Nirgends konnte und wollte ich das Lehrgebäude als fertig darstellen."

Diese Worte aus der Vorrede offenbaren den Geist des ganzen Unternehmens, und was vorliegt, zeigt, daß diesem Geiste gemäß gehandelt warde. Die Abschnitte über die Physik und Chemie des Lebendigen sind außerordentlich reich und trotz der Fülle des Gebotenen sehr klar und übersichtlich. Der Referent bekennt gern, sie mit sehr großem Nutzen für sich studiert zu haben. Überall finden sich schon Hinweise anf die spätere biologische Verwertung. Stets ist auf die Originalliteratur zuriickgegangen, die der Verfasser offenbar in ganz erstaunlichem Maße beherrscht.

Etwas mehr möge über das erste Kapitel >Allgemeine Charakteristik des Lebens gesagt sein. Gerade dieser Abschnitt stellt das Werk in die Nähe jener älteren Physiologien eines Treviranus, Burdach, Johannes Müller, in denen Physiologie als integrierender Teil des menschlichen Wissens als eines Ganzen behandelt worden war.

Sehr klar sind die Abschnitte über Stoff- und Energiewechsel; überall ist Begriffsklarheit das erste, was erstrebt wird. Gute Schemata, z. B. uiber die Speicherung interatomarer und intermolekularer Energie, erleichtern das Verständnis.

Anorganische Analogien zu Lebensvorgängen werden kritisch und mit großer Vorsicht erörtert; nur s die Parallelisierung der Endvorgänge lebenden Substanz "mit wohlstudierten gesetzmäßigen Umsetzungen am unbelebten Stoff hat swertvolle Aufschlüsse geliefert* (S. 35). 
Der vierte Abschnitt des ersten Kapitels "Antonomie des Belebten, Dualitait von Belebtem und Unbelebtem \& bezeichnet seinen Höhepunkt. >Die Selbstregulierung der Erregbarkeits, die sich in der "Adaptation an längerdauernde Reize* zeigt (S. 38), wird besonders scharf betont. Die Entwicklungsphysiologie kommt schon hier voll zn ihrem Recht.

In dem Abschnitt Naturphilosophische Lebenstheorien « wird das vitalistische Problem mit wirklicher Sachkenntnis (was nicht eben häufig ist) erörtert. Man merkt das an Kleinigkeiten, z. B. daran, daß der Verfasser mit Rücksicht auf die Lehren des Referenten ausdrücklich betont, es werde der Entelechie skeinerlei Leistung aufgebiirdet, welche an sich irgend einen Energiebeitrag reprïsentierte (S. 51). Sachlich entschieden wird vom Verfasser der Streit zwischen Vitalismus und Mechanismus nicht: "Wir betrachten die Physiologie nicht einfach als angewandte Physik und Chemie, vielmebr das Leben als einen Erscheinungskomplex für sich, Belebtes and Unbelebtes als verschiedene, selbständige und gleichwertige Objekte der naturwissenschaftlichen Forschung.* Mit diesem sphänomenologischen Dualismus muß sich sdie exakte Physiologie, als pbänomenalistische Naturwissenschaft begnügen* (S. 43). Eine Erörterung des Problems der Urzeugung schließt das erste Kapitel.

Aus dem zweiten und dritten Kapitel sei noch ausdriicklich auf die $>$ Lehre von der Formart" (Kolloidchemie) (S. $70 \mathrm{ff}$.) und auf die allgemeine Kennzeichnung der sChemischen Natur der lebenden Substanz (S. 158ff.) hingewiesen. Übrigens will der Verfasser unter slebender Substanze (gleich Roux) nur seine funktionelle, nicht notwendig auch chemische Einheit verstehen (S. 161).

Mit großer Spannung wird jeder, der den vorliegenden Halbband von Tschermaks "Allgemeiner Physiologie" kennen gelernt hat, seine hoffentlich recht bald erscheinenden Fortsetzungen erwarten.

Nicht nur jeder Biologe sollte dieses Werk studieren, sondern auch jeder Philosoph, der über naturphilosophische Dinge sachlich und wahrhaft s kritisch *, und nicht, wie die meisten Neukantianer das tun, dogmatisch mitreden will.

Gustav Störring, Logik. Leipzig, Wilhelm Engelmann. 1916. VIII u. 363 s. Preis M. 12.-

Logische und erkenntnistheoretische Werke, die von Männern geschrieben sind, welche ursprünglich Naturforscher oder Psychologen waren, sind geradezu ein Kennzeichen der neuesten Philosophie. Wir haben die Werke von WundT, Mach, Ostwald, Külpe, Zienen, v. Kries und des Referenten sOrdnungslehrea, und wir haben in Störrings nenestem Werke nun wiederum eine "Logike, die von einem ursprüngliehen Psychologen stammt. Einen Mann mit solcher Herkunft zum Verfasser zu haben, ist stets von vornherein ein Vorzug eines philosophischen Werkes, denn gründliche Kenntnisse auf einem Sondergebiete zu haben, ist eben ein Vorzug; auch darf man von einem Autor, der gleichsam durch Berufsänderung zur Philosophie kommt, stets sagen, daß er ein ganz besonders starkes Bewußtsein von der Notwendigkeit der Philosophie besitze.

Der Natur dieser Zeitschrift entsprechend kann STörRINGs Werk hier nur kurz angezeigt werden. Es ist ein richtiges Lehrbuch von handlichem Umfange. Die Darstellung ist sehr klar und leichtverständlich; anch die angeblich strockenen * Teile der Logik, wie die Lehre von der Umwandlung der Urteile und vom Schlusse, 
lesen sich fesselnd und anregend. Ein besonderer Vorzug sind die vielen historischen nnd kritischen Abschnitte. Der Leser bekommt durch sie ein anschauliches Bild vom gesamten Stande einer Frage und vom jeweiligen Widerstreit der Meinungen. Ein anderer Vorzug sind die Exkurse in die Psychologie des Denkens, welche mit Recht stets scharf vom eigentlich Logischen getrennt sind.

Den Naturforscher wird-zumal die sMethodenlehre im speziellen Sinnes interessieren; hier wird auf Erörterungen von KirchHoff, O. HerTwig, Roux, Verworn u. a. Bezug genommen. Mit Recht wird die von Verworn gelehrte »Gleichwertigkeit der ein Geschehnis bedingenden Faktoren bestritten (S. 316); andere Seiten der STörrivgschen Kausalitätslehre kann der Referent freilich nicht annehmen, z. B. daß es seine mystische Betrachtungsweise sei, wenn Roux von einem > Hervorgebrachtwerden < der Wirkung durch die Ursache redet (S. 315). Leider fehlt dem Werk eine eingehende Erörterung des Begriffs Entwicklung.

Auf S. 319 findet sich in bezug anf die Lehren des Referenten ein, von VerworN uibernommener, Irrtum. Ich soll, durch meine Begriffe sprospektive Potenz * und »indeterminiert-äquipotentielles System ‘, den Indeterminismus überhaupt gelehrt haben. Ich habe aber niemals den Indeterminismus des biologischen Geschehens gelehrt, ich habe vielmehr die Gültigkeit des Begriffs der eindeutigen Bestimmtheit anch für meinen Vitalismus sehr streng festgehalten (s. Phil. d. Org. II. S. 1503ff.). Wenn ich (Arch. f. Entw.-Mech. VIII. 1899. S. 73) den Ausdruck sindeterminiert-äquipotentielles System e einmal 1) verwendete, so soll dieser nur eine kurze Bezeichnung für den experimentell bewiesenen Sachverbalt sein, daß die einzelnen Zellen eines mit jenem Namen gekennzeichneten Systems nicht fest für bestimmte Einzelleistangen determiniert seien, also dafür, $\mathrm{da} B$ ihre morphologische Leistungsfaihigkeit keine fest vou vornherein eingeengte ist. Aber das Gesehehen überhaupt an den in Frage kommenden Systemen gilt mir als eindeutig bestimmt, freilich durch die materielle Konstellation und durch Entelechie bestimmt.

Schließen wir mit dem Urteil, daß Störrings Werk sehr zum Studinm zu empfehlen ist, zumal für die erste gründliche Einführung in den Gegenstand.

1) In der sPhilosophie des Organischen (I. S. 122) steht übrigens für sindeterminiert-äquipotentiell > unbeschränkt-aquipotentiell ; es handelt sich um einen Durchgangsbegriff, dessen Verfolgung schließlich zum Begriff sharmonisch-äquipotentielles System* fiihrt. 